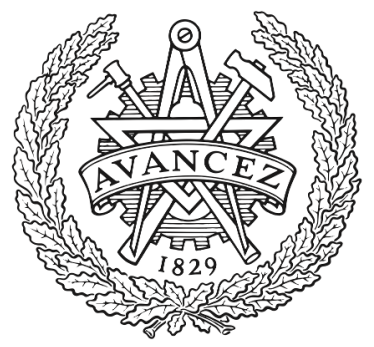

CHALMERS

UNIVERSITY OF TECHNOLOGY

\title{
Improved Pedestrian Detection under Mutual Interference by FMCW Radar Communications
}

Downloaded from: https://research.chalmers.se, 2023-04-26 05:22 UTC

Citation for the original published paper (version of record):

Aydogdu, C., Garcia, N., Wymeersch, H. (2018). Improved Pedestrian Detection under Mutual Interference by FMCW Radar Communications. IEEE International Symposium on Personal, Indoor and Mobile Radio Communications, PIMRC: 101-105.

http://dx.doi.org/10.1109/PIMRC.2018.8581028

N.B. When citing this work, cite the original published paper. 


\title{
Improved Pedestrian Detection under Mutual Interference by FMCW Radar Communications
}

\author{
Canan Aydogdu, Nil Garcia, and Henk Wymeersch \\ Department of Electrical Engineering \\ Chalmers University of Technology, Sweden \\ e-mail: canan@chalmers.se
}

\begin{abstract}
The widespread of automotive radars leads to increase of mutual interference, which in turn degrades road safety. The effect of mutual interference with a focus on detection of pedestrians is investigated. It is shown that detection of pedestrians degrades in the presence of mutual interference. A joint radar communication solution is proposed that increases pedestrian detection probability with negligible impact in the ranging error.
\end{abstract}

\section{INTRODUCTION}

Pedestrian traffic fatalities have been rising at alarming rates: in the U.S. the number has reached 6000 [1], which is expressed to be the highest in two decades, while in Europe, 5435 pedestrians were killed in 2015, which is $20 \%$ of all road fatalities [2]. In order to improve pedestrian safety, automotive radar sensors should be designed to accurately detect and track pedestrians, even in bad weather [3]. The small radar cross section (RCS) of the pedestrians and the clutter make pedestrian detection challenging. Two main tracks exist in the literature to mitigate the pedestrian detection problem: (i) improving the characterization of reflected signals by pedestrians by measurements [4]-[8] and (ii) radar signal processing for better detection [9]-[14].

As frequency modulated continuous wave (FMCW) radars become more widespread (with multiple radars per vehicle), the mutual interference between radars becomes the critical factor that reduces their detection capability [15], thereby affecting detection of small targets such as pedestrians [16]. One way to eliminate the mutual interference is to coordinate radar sensors through communication, either via a separate link (e.g., 802.11p) or via the radar transceiver itself, i.e., radar communication (RadCom). The combination of communication and radar has been proposed a number of times in various forms and applications [17], [18]. Most works consider orthogonal frequency division multiplexing (OFDM) for joint radar communications [19]-[23]. OFDM is widely used in communication due to its high degree of flexibility, low receiver complexity, and high performance under different propagation conditions [24]-[27]. However, due to the low-rate analog-to-digital convertor (ADCs) of FMCW radars, OFDM cannot fully occupy the radar band (77-81 $\mathrm{GHz}$ ), limiting its applicability.

In this paper, we extend our proposal for a highresolution FMCW radar based RadCom from [28] to account for the effect of pedestrians in the environment. This method uses a small part of the radar bandwidth to create a vehicle-to-vehicle (V2V) connection, which

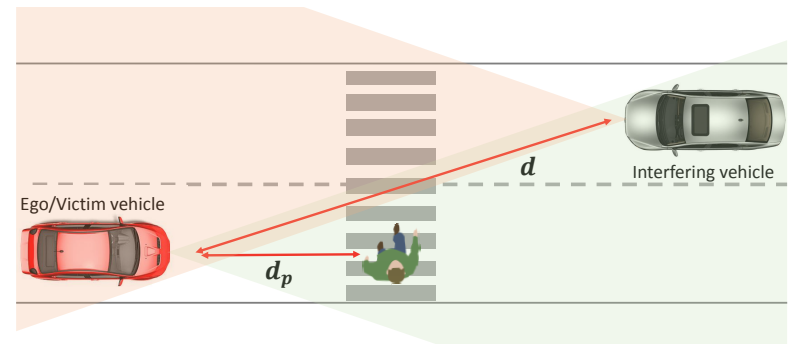

Fig. 1. Scenario where pedestrian detection is obscured with two vehicles in each other's field of view leading to mutual interference.

is controlled via a carrier sense multiple access (CSMA) protocol and is utilized to control the timing of radar signals. We have performed a simulation of the proposed concept for a two-vehicle and one pedestrian scenario and found that, under realistic propagation conditions, RadCom can significantly reduce the radar interference and increase pedestrian detection probability with negligible performance degradation in terms of radar accuracy.

\section{SySTEM MODEL}

We focus on the scenario shown in Fig. 1, consisting of an ego/victim vehicle, an interfering vehicle and a pedestrian. The interfering vehicle is located at a distance $d$ from the ego vehicle and the pedestrian is facing the ego vehicle located at a distance $d_{p}$ from the ego vehicle. Parameter $v$ denotes the relative ${ }^{1}$ velocity between the vehicles, while $v_{p}$ is the relative velocity between the ego vehicle and the pedestrian.

\section{A. FMCW Transmitter}

We consider a sequence of frequency modulated continuous waves, i.e., chirps, transmitted by an FMCW radar, of the form

$$
s(t)=\sqrt{P_{\mathrm{tx}}} \sum_{k=1}^{N} c(t-k T),
$$

where $c(t)$ is a chirp of the form

$$
c(t)=\exp \left[j 2 \pi\left(f_{c}+\frac{B}{T} t\right) t\right],
$$

where $P_{\mathrm{tx}}$ is the transmit power, $B$ denotes the radar bandwidth (typically $1-4 \mathrm{GHz}$ ), $f_{c}$ is the carrier frequency

\footnotetext{
${ }^{1} \mathrm{~A}$ positive $v$ corresponds to approaching vehicles and a positive Doppler shift, which leads to a decreased time difference between the transmitted and reflected radar signal.
} 
(77 GHz), $T$ is the chirp duration, and $N$ is the number of chirps per frame. The frame time $T_{f}$ comprises $N T$ plus the idle and processing time. Chirp parameters are designed to meet the maximum detectable range $\left(d_{\max }\right)$, maximum detectable relative velocity $\left(v_{\max }\right)$, the range and velocity resolution requirements of an automotive radar.

\section{B. FMCW Receiver}

At the co-located receiver, the backscattered signal is processed. The radar receiver comprises of a mixer, an ADC, and a digital processor. The mixer multiplies the received signal with a copy of the transmitted chirp. After low-pass filtering the resulting intermediate frequency (IF) signal, the mixer will output a signal with multiple harmonics at frequencies proportional to the time difference between the transmitted chirp and the received chirps. The output of the mixer is then sampled by the ADC, with sampling interval $T_{s}$, and passed to the digital processor which will detect and estimate the frequencies. The ADC bandwidth $1 /\left(2 T_{s}\right)$ is generally on the order of $10-50 \mathrm{MHz}$ and is thus much smaller than $B$. In the absence of interference, sample $n$ of chirp $k$ of the back-scatter signal at the ego vehicle is of the form [3]

$$
\begin{aligned}
& r_{n}^{(k)}= \\
& \sqrt{\gamma P_{\mathrm{tx}} d^{-4}} \exp \left(j 2 \pi \frac{B(2 d / c-2 \beta)}{T} n T_{s}\right)+w_{n}^{(k)} \\
& +\sqrt{\gamma_{p} P_{\mathrm{tx}} d_{p}^{-4}} \exp \left(j 2 \pi \frac{B\left(2 d_{p} / c-2 \beta_{p}\right)}{T} n T_{s}\right)
\end{aligned}
$$

where the first term corresponds to the reflected off signal from the vehicle and the second term to the reflected off signal from the pedestrian; and $\gamma=G_{\mathrm{tx}} G_{\mathrm{rx}} \sigma \lambda^{2} /(4 \pi)^{3}$ for vehicle RCS $\sigma_{c}, \gamma_{p}=G_{\mathrm{tx}} G_{\mathrm{rx}} \sigma_{p} \lambda^{2} /(4 \pi)^{3}$ for pedestrian RCS $\sigma_{p}$, transmitter and receiver antenna gains $G_{\mathrm{tx}}$ and $G_{\mathrm{rx}}$, Doppler time shift between ego and interfering vehicle $\beta=T v f_{c} /(B c)$, Doppler time shift between ego vehicle and the pedestrian $\beta_{p}=T v_{p} f_{c} /(B c)$, in which $c$ denotes the speed of light, $w_{n}^{(k)}$ is additive white Gaussian noise (AWGN) with variance $N_{0}$.

A common approach to frequency retrieval in FMCW radar is to compute the fast Fourier transform (FFT) of the signal, average the signal through multiple chirp periods for enhanced SNR, and detect the peaks in the frequency-domain.

\section{Goal}

Our aim is to study the pedestrian detection performance under the presence of mutual interference. Precisely, we investigate how the probability of pedestrian detection, vulnerable period and ranging error are affected by the proposed FMCW-based RadCom system in [28].

\section{ANALYSIS OF PEDESTRIAN \\ DETECTION UNDER MUtUAL RADAR INTERFERENCE}

In this section, we describe the interference model in presence of pedestrians and calculate the conditions under which interference exists.

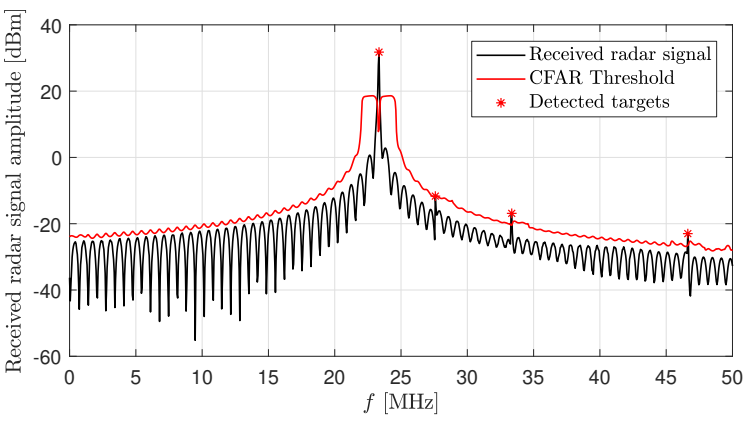

Fig. 2. FFT of received signal $\left[\tilde{r}_{n}^{(k)}\right]_{n=1}^{T / T_{s}}$, with a interfering vehicle target and a pedestrian target. Four peaks are visible: two real targets and two targets due to interference.

\section{A. Interference Model}

In the scenario in Fig. 1, both radars are FMCW based and use the same frequency band. If the interfering vehicle transmits its FMCW signal with a delay $\tau$ with respect to the ego vehicle, the received signal at the ego radar becomes $\tilde{r}_{n}^{(k)}=r_{n}^{(k)}+I_{n}^{(k)}$, where the interfering signal $I_{n}^{(k)}$ is received at the ego radar at time $\tau+d / c-\beta$ when travelling through the direct path and at $\left(\tau+\left(d_{p}+\right.\right.$ $\left.\left.d_{\mathrm{vp}}\right) / c-\beta p-\beta_{\mathrm{vp}}\right)$ when travelling via the pedestrian:

$I_{n}^{(k)}=\sqrt{P_{\mathrm{tx}}} \times$

$\begin{cases}0 & \tau \notin V \\ \sqrt{\tilde{\gamma} d^{-2}} e^{j 2 \pi \frac{B\left(\tau+d / c-\tau_{D}\right)}{T} n T_{s}} & \tau \in V \\ +\sqrt{\gamma\left(d_{p} d_{\mathrm{vp}}\right)^{-2}} e^{j 2 \pi \frac{B\left(\tau+\left(d_{p}+d_{\mathrm{vp}}\right) / c-\beta p-\beta_{\mathrm{vp}}\right)}{T} n T_{s}} . & \end{cases}$

Above, $\tilde{\gamma}=G_{\mathrm{tx}} G_{\mathrm{rx}} \lambda^{2} /(4 \pi)^{2}$ and $V$ is the so-called vulnerable period, defined as the time interval during which received signals from facing radars cause mutual interference. If the radar transmits chirps within the vulnerable period, a direct and a reflected interference is caused. The vulnerable period was introduced and calculated in [28] but neglected interference reflected from obstacles. In this study, we extend this result by taking into account pedestrian reflection.

Example 1. Fig. 2 shows an example of a received FMCW signal, where the ego vehicle is located at $[0,0]$, interfering vehicle at $[126 \mathrm{~m}, 60 \mathrm{~m}]$, i.e., $d=140 \mathrm{~m}$, and pedestrian at $[100 \mathrm{~m}, 0]$ for relative vehicle speed of $60 \mathrm{~km} / \mathrm{h}$ (approaching), RCS of $\sigma_{p}=1 \mathrm{~m}^{2}$ and CFAR threshold of $5 \mathrm{dBm}$. The chirp transmissions have a starting time difference of $\tau=0$, meaning the radars transmit within the vulnerable period and cause interference. The pedestrian and the vehicles are placed so that the direct and pedestrian reflected interference signals have a higher time of flight difference, which makes observation of the two distinct interference signals possible. Under these conditions, the direct interference, the-pedestrian-reflected interference, the pedestrian and the vehicle are detected as shown in Fig. 2. Note that it is generally hard to distinguish between direct and pedestrian-reflecting-interference for the scenario in Fig. 1 or lower pedestrian RCS. 


\section{B. Interference Condition}

The ego vehicle starts an FMCW transmission at time $t=0$ and the interfering vehicle starts a transmission at time $t=\tau$. We will now determine the vulnerable period for different conditions.

- Without Doppler: The interfering transmission arrives at the ego vehicle directly at time $t^{\prime}=\tau+d / c$ and through pedestrian reflection at time $t^{\prime}=\tau+\left(d_{p}+d_{\mathrm{vp}}\right) / c$. Interference will occur when $t^{\prime} \in\left[0, T /\left(2 B T_{s}\right)\right]$. Assuming that the interfering vehicle and the pedestrian are within $d_{\max }$, the maximum possible propagation delay of both the direct and pedestrian reflected path is $\max \left\{d_{p}+d_{\mathrm{vp}}\right\}=3 d_{\max } / c$. Since $d_{\max }$ is the maximum detectable radar range and is related to chirp parameters by $d_{\max }=c T /\left(4 B T_{s}\right)$ [29], the vulnerable period for the ego vehicle corresponds to all transmission times $t^{\prime}$ of other vehicles for which

$$
\tau \in V=\left[-\frac{3 T}{4 B T_{s}}, \frac{T}{2 B T_{s}}\right] .
$$

- With Doppler: Given that the pedestrian and vehicle have relative speeds with respect to the ego vehicle smaller than $v_{\max }$, which is the maximum detectable relative velocity (approaching or receding), the maximum time shift due to doppler can be $T v_{\max } f_{c} / B c$ for the direct interference and $3 T v_{\max } f_{c} / B c$ for the pedestrian-reflectedinterference. Taking the maximum of these shifts and substituting $v_{\max }=c /\left(4 f_{c} T\right)$ [29], the vulnerable period for a transmission at time $t=0$ becomes

$$
V=\left[-\frac{3 T}{4 B T_{s}}-\frac{3}{4 B}, \frac{T}{2 B T_{s}}+\frac{3}{4 B}\right] .
$$

Since $T_{s} \ll T$, the Doppler time shift is negligible and it turns out that the vulnerable period computed taking the pedestrian reflecting interference signal into account is, under ideal low-pass filtering, given by (5).

Given the computed vulnerable period, the transmission times of FMCW radar chirps of other vehicles can thus be coordinated to ensure that $\tau \notin V$. Our proposed RadCom approach [28] avoids mutual interference by assigning different radars to non-overlapping vulnerable periods through TDMA (rTDMA), whereas this coordination among vehicles is provided through CSMA-based communications (cCSMA) taking place in a reserved fraction of spectrum $B_{c}$ of the full bandwidth $B$. Hence, we propose a RadCom scheme sharing the medium through FDM/rTDMA/cCSMA, where the reader is referred to [28] for full details.

Remark 2. Reception of negative edge frequencies of the imperfect low-pass filtering at the ADC can also cause mutual interference, especially for the direct interference signal at small $d$, which decays by $d^{2}$ and is still high enough to reside after low-pass filtering. However, the pedestrian reflected interference signal power decays by $d_{p}^{2} d_{\mathrm{vp}}^{2}$ and has no negative frequency components after low-pass filtering. Hence the vulnerable period
TABLE I

SIMULATION PARAMETERS.

\begin{tabular}{ll}
\hline Parameter & Value \\
\hline Vehicle RCS $\left(\sigma_{c}\right)$ & $20 \mathrm{~m}^{2}$ \\
Pedestrian RCS $\left(\sigma_{p}\right)$ & $0.3162 \mathrm{~m}^{2}$ \\
Chirp duration $(T)$ & $20 \mu \mathrm{s}$ \\
Frame duration $\left(T_{f}\right)$ & $20 \mathrm{~ms}$ \\
Time slots per frame $(K)$ & 10 \\
Radar bandwidth & $0.96 \mathrm{GHz}-1 \mathrm{GHz}$ \\
$d_{\text {max }}$ & $150 \mathrm{~m}$ \\
$v_{\text {max }}$ & $140 \mathrm{~km} / \mathrm{h}$ \\
$v$ & $100 \mathrm{~km} / \mathrm{h}$ \\
$P_{\text {tx }}$ & $1 \mathrm{~W}$ \\
$N$ & 99 \\
$f_{c}$ & 77 \\
$T_{s}$ & $0.01 \mu \mathrm{s}$ \\
Chebyshev low-pass filter order & 13 \\
Thermal noise temperature & $290 \mathrm{~K}$ \\
Receiver's noise figure & $4.5 \mathrm{~dB}$ \\
\hline \multirow{\Xi}{\Xi}{ Communication bandwidth $B_{c}$} & $20 \mathrm{MHz}, 40 \mathrm{MHz}$ \\
Packet size & $600 \mathrm{Bytes}$ \\
Modulation & $16-\mathrm{QAM}$ \\
MAC & $1 / 2-$ persistent CSMA \\
Maximum contention window & 6 \\
\hline
\end{tabular}

can be extended to $V=T /\left(B T_{s}\right)[-1,1 / 2]$, which is approximately equal to the vulnerable period computed with no obstacles or pedestrians from [28].

\section{Performance Evaluation and Results}

The performance of pedestrian detection by an FMCW receiver in presence of mutual interference from an FMCW automotive radar is investigated by Matlab $2017 \mathrm{~b}$ simulations, which use the computed vulnerable period for RadCom. And a comparison is made by the proposed FMCW-based RadCom system in terms of the pedestrian detection probability and the ranging error. The performance of radar without mutual interference is the same as RadCom case. The probability of mutual interference and the SNR of pedestrians are also evaluated.

\section{A. Simulation Parameters}

The ego vehicle is located at $[0,0]$, the interfering vehicle at $\left[\sqrt{d^{2}-9 \mathrm{~m}}, 3 \mathrm{~m}\right]$ and the pedestrian at $\left[d_{p}, 0\right]$. The vehicles are assumed to move towards each other with relative speed $v=100 \mathrm{~km} / \mathrm{h}$. The simulation parameters are summarized in Table I. Two facing vehicles are assumed to have radars with the same properties, with $\tau$ fixed to 0 . Radar is FMCW with sawtooth waveform. The chirp sequence is designed so as to meet the maximum detectable relative velocity $v_{\max }=140 \mathrm{~km} / \mathrm{h}$, the maximum detectable range $d_{\max }=150 \mathrm{~m}$, velocity resolution smaller than $1 \mathrm{~m} / \mathrm{s}$ and range resolution of $15 \mathrm{~cm}$. Radar front-end-hardware component parameters are taken as in [30]. The mean value for the radar cross section of a car is taken as $20 \mathrm{~m}^{2}$ [30], [31]. The pedestrian RCS at $77 \mathrm{GHz}$ is set to $-5 \mathrm{~dB} / \mathrm{m}^{2}$ [7]. At the signal processing stage, the received and transmitted chirp sequences are mixed, passed from the ADC with Chebyshew low-pass filters of order 13. GoCA-CFAR thresholding with 50 training cells with 2 guard cells is used for radar detection. 
The RadCom system has a communication bandwidth of $B_{c}=40 \mathrm{MHz}$, leaving $B-B_{c}$ for the radar.

The performance is evaluated in terms of the pedestrian detection probability $P_{d}$, ranging error of the pedestrian and SNR of the signal reflected by the pedestrian and other interference. We note (results not shown) that probability of false alarm was 1 without RadCom and close to zero with RadCom, similar to [28]. In Fig. 1, 100 Monte Carlo simulations were conducted with the two vehicles exposed to mutual interference.

\section{B. Results}

1) Pedestrian Detection Probability: The pedestrian detection probability as a function of $d_{p}$ is plotted in Fig. 3 for a fixed $d=50 \mathrm{~m}$. In the radar-only case, we observe that the pedestrian goes undetected for most values of $d_{p}$. In particular, the pedestrian is not detected when its location is approximately the same than the ghost target location $(d / 2=$ $25 \mathrm{~m}$ for this case), due to the mutual interference. When the interfering vehicle and the pedestrians are close but far away from the ego vehicle, the probability of detection is zero due to the increased noise floor caused by the interference. On the other hand, with the RadCom system, $P_{d}=1$ because the radar signals do not collide thanks to the $\mathrm{V} 2 \mathrm{~V}$ communication, except for two points: i) $d_{p}=150 \mathrm{~m}$ when the signal reflected by the the pedestrian becomes too weak, and ii) $d_{p}=d$ which corresponds to the case where the vehicle and pedestrian are located at the same range.

Fig. 4 shows $P_{d}$ for varying separation distance between the two vehicles $d$ while the pedestrian distance is kept fixed at $d_{p}=50 \mathrm{~m}$. Again, the pedestrian detection probability drops to zero for the radar only case when the range of the ghost target (interference) is around $50 \mathrm{~m}$ (where the pedestrian is located). While $P_{d}=1$ with RadCom, except for $d_{p}=d$ when the vehicle and pedestrian are located at the same range. Note that $P_{d}=1$ when $d=150 \mathrm{~m}$, since the reflected signal from the pedestrian located at $d=50$ $\mathrm{m}$ is strong enough to be detected.

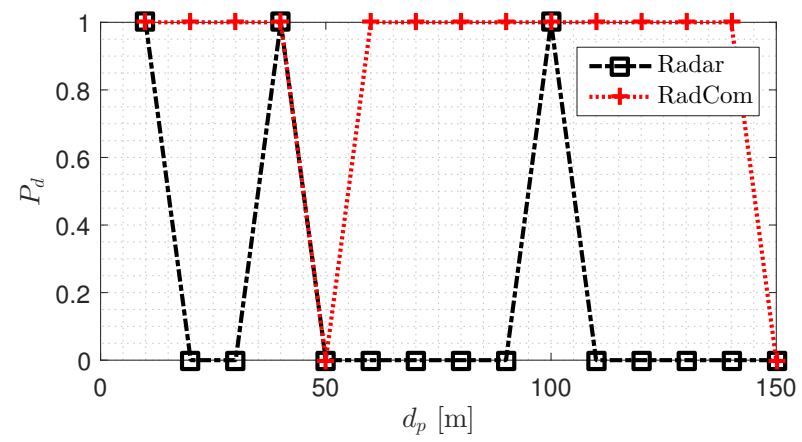

Fig. 3. Pedestrian detection probability for varying vehicle and pedestrian separation distance $d_{p}$.

2) $S N R$ : The pedestrian detection probability is closely coupled to SNR of the signal reflected by the pedestrian, and the SNR of the signal coming in a direct path from the interfering vehicle plus the ghost targets. These SNR values are compared for the radar-only scheme and RadCom in

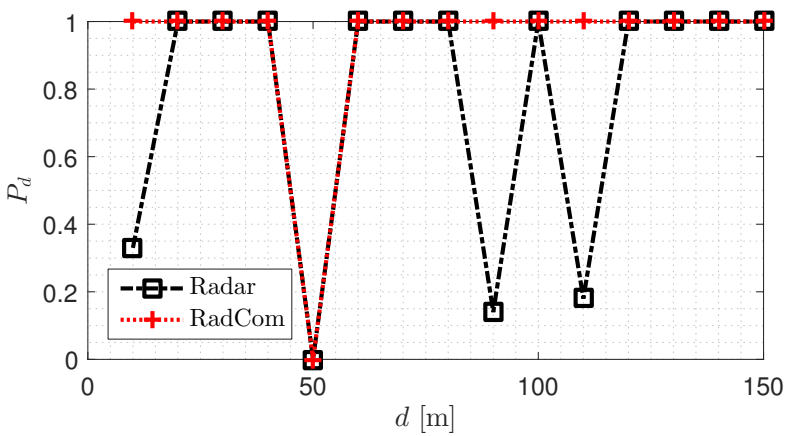

Fig. 4. Pedestrian detection probability varying distance between vehicles $d$.

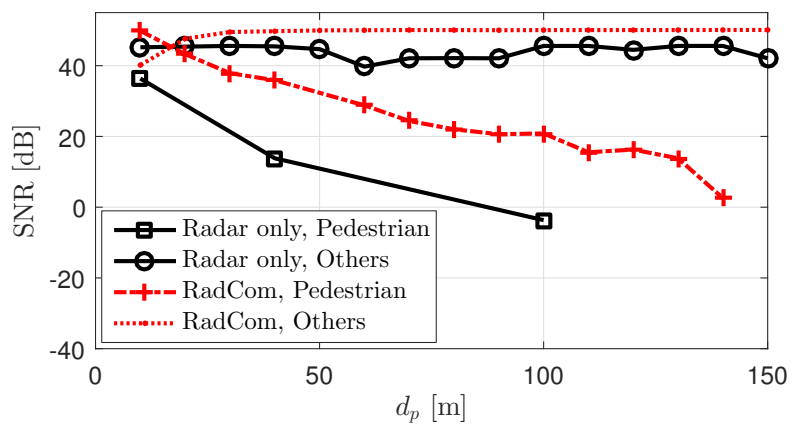

Fig. 5. SNR of pedestrian and other detections (interfering vehicle plus ghost targets) for radar only scheme and RadCom for varying vehicle and pedestrian separation distance $d_{p}$.

Fig. 5 for a fixed interfering vehicle distance $d=50 \mathrm{~m}$ and varying pedestrian distance $d_{p}$. It is observed that the SNR of other targets is considerably higher than the pedestrian SNR, except for $d_{p}=10 \mathrm{~m}$ in the case of RadCom, where the pedestrian is very close to the ego vehicle. With RadCom, the pedestrian SNR improves considerably and provides detection of pedestrians for $d_{p} \leq 110 \mathrm{~m}$. RadCom also increases the SNR of the other vehicle. Note that the pedestrian SNR naturally decreases with $d_{p}$.

3) Ranging Error: Fig. 6 shows the ranging error of the pedestrian with and without RadCom, considering different $d_{p}$. The ranging error is observed to be below $10 \mathrm{~cm}$. RadCom has slightly worse accuracy due to the reduced bandwidth, but this effect is quite small.

\section{CONCLUSION}

Automotive radars can interfere with each other due to the presence of pedestrians and other objects, creating ghost targets and negatively affecting road safety. We have quantified under which conditions ghost targets occur and evaluated a RadCom scheme which reduces interference by adjusting the radar time over a dedicated $\mathrm{V} 2 \mathrm{~V}$ band, while reusing the radar hardware for communication. By time multiplexing radar transmissions of FMCW automotive radars, we are able to mitigate radar interference and increase pedestrian detection probability without impacting the pedestrian ranging accuracy. Performance in terms of detection probability, SNR, and ranging accuracy are 


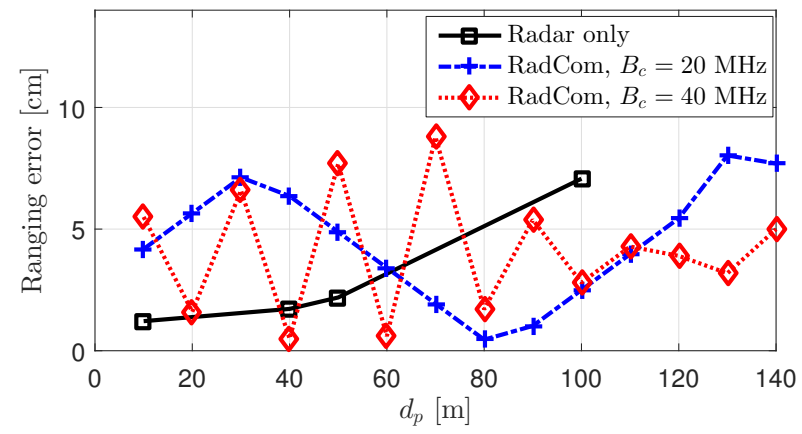

Fig. 6. Comparison of ranging error without and with RadCom for $d=50 \mathrm{~m}$.

reported, based on high-fidelity simulations. Future work will consider larger-scale scenarios with multiple pedestrians as well as the interference from multipath. Although a hardware implementation of the proposed scheme is not currently available, we plan to implement it in the future.

\section{ACKNOWLEDGMENT}

This work was supported, in part, by Marie Curie Individual Fellowships (H2020-MSCA-IF-2016), Grant 745706 (GreenLoc), and a SEED grant from Electrical Engineering Department of Chalmers University of Technology.

\section{REFERENCES}

[1] R. Retting, "Final report, pedestrian traffic fatalities by state: 2016 preliminary data," 2017. [Online]. Available: https://www. ghsa.org/sites/default/files/2017-03/2017ped_FINAL_4.pdf

[2] E. Commision, "Traffic safety basic facts 2017," 2017. [Online]. Available: https://ec.europa.eu/transport/road_safety/sites/ roadsafety/files/pdf/statistics/dacota/bfs20xx_pedestrians.pdf

[3] S. M. Patole, M. Torlak, D. Wang, and M. Ali, "Automotive radars: A review of signal processing techniques," IEEE Signal Processing Magazine, vol. 34, no. 2, pp. 22-35, 2017.

[4] H. Ritter and H. Rohling, "Pedestrian detection based on automotive radar," in IET International Conference on Radar Systems, Oct 2007, pp. 1-4.

[5] E. Marchetti, R. Du, F. Norouzian, E. G. Hoare, T. Y. Tran, M. Cherniakov, and M. Gashinova, "Comparison of pedestrian reflectivities at 24 and $300 \mathrm{GHz}$," in 18th International Radar Symposium (IRS), June 2017, pp. 1-7.

[6] M. Chen and C. C. Chen, "RCS patterns of pedestrians at 76-77 GHz," IEEE Antennas and Propagation Magazine, vol. 56, no. 4, pp. 252-263, Aug 2014.

[7] D. Belgiovane, C. C. Chen, M. Chen, S. Y. P. Chien, and R. Sherony, "77 GHz radar scattering properties of pedestrians," in IEEE Radar Conference, May 2014, pp. 0735-0738.

[8] D. Belgiovane and C. C. Chen, "Bicycles and human riders backscattering at $77 \mathrm{GHz}$ for automotive radar," in 10th European Conference on Antennas and Propagation (EuCAP), April 2016, pp. 1-5.

[9] D. T. Linzmeier, M. Skutek, M. Mekhaiel, and K. C. J. Dietmayer, "A pedestrian detection system based on thermopile and radar sensor data fusion," in 7th International Conference on Information Fusion, vol. 2, July 2005, pp. 1272-1279.

[10] H. Rohling, S. Heuel, and H. Ritter, "Pedestrian detection procedure integrated into an $24 \mathrm{GHz}$ automotive radar," in IEEE Radar Conference, May 2010, pp. 1229-1232.

[11] K. Thurn, M. Heuer, G. Li, S. Max, M. M. Meinecke, and M. Vossiek, "Pedestrian detection with an interlaced chirp sequence concept in automotive radar," in 2015 16th International Radar Symposium (IRS), June 2015, pp. 161-166.

[12] M. Yasugi, Y. Cao, K. Kobayashi, T. Morita, T. Kishigami, and Y. Nakagawa, "79 GHz-band radar cross section measurement for pedestrian detection," in 2013 Asia-Pacific Microwave Conference Proceedings (APMC), Nov 2013, pp. 576-578.
[13] T. Kishigami, K. Kobayashi, M. Otani, T. Morita, H. Mukai, A. Saito, and Y. Nakagawa, "Advanced millimeter-wave radar system using coded pulse compression and adaptive array for pedestrian detection," in IEEE Radar Conference (RadarCon13), April 2013, pp. 1-6.

[14] Y. Okamoto, I. Matsunami, and A. Kajiwara, "Pedestrian and twowheeler detection using ultra-wideband vehicular radar," in IEEE Sensors Applications Symposium Proceedings, Feb 2012, pp. 1-4.

[15] I. M. Kunert, "Project final report, MOSARIM: More safety for all by radar interference mitigation," 2012. [Online]. Available: http://cordis.europa.eu/docs/projects/cnect/1/248231/ 080/deliverables/001-D611finalreportfinal.pdf

[16] C. Fischer, M. Barjenbruch, H. L. Bloecher, and W. Menzel, "Detection of pedestrians in road environments with mutual interference," in 14th International Radar Symposium (IRS), vol. 2, June 2013, pp. 746-751.

[17] R. Cager, D. LaFlame, and L. Parode, "Orbiter ku-band integrated radar and communications subsystem," IEEE Transactions on Communications, vol. 26, no. 11, pp. 1604-1619, November 1978.

[18] W. Wiesbeck, "SDRS: software-defined radar sensors," in IGARSS 2001. Scanning the Present and Resolving the Future. Proceedings. IEEE 2001 International Geoscience and Remote Sensing Symposium (Cat. No.01CH37217), vol. 7, 2001, pp. 3259-3261 vol.7.

[19] B. J. Donnet and I. D. Longstaff, "Combining MIMO radar with OFDM communications," in 2006 European Radar Conference, Sept 2006, pp. 37-40.

[20] D. Garmatyuk, J. Schuerger, and K. Kauffman, "Multifunctional software-defined radar sensor and data communication system," IEEE Sensors Journal, vol. 11, no. 1, pp. 99-106, Jan 2011.

[21] C. Sturm and W. Wiesbeck, "Waveform design and signal processing aspects for fusion of wireless communications and radar sensing," Proceedings of the IEEE, vol. 99, no. 7, pp. 1236-1259, July 2011.

[22] P. Kumari, N. Gonzalez-Prelcic, and R. W. Heath, "Investigating the IEEE 802.11ad standard for millimeter wave automotive radar," in 2015 IEEE 82nd Vehicular Technology Conference (VTC2015-Fall), Sept 2015, pp. 1-5.

[23] J. Choi, V. Va, N. Gonzalez-Prelcic, R. Daniels, C. R. Bhat, and R. W. Heath, "Millimeter-wave vehicular communication to support massive automotive sensing," IEEE Communications Magazine, vol. 54, no. 12, pp. 160-167, December 2016.

[24] B. Nuss, "Multi-user MIMO-OFDM radar with simultaneous communication," German Research Foundation (DFG) and Karlsruhe Institute of Technology (KIT), 2007.

[25] P. Falcone, F. Colone, C. Bongioanni, and P. Lombardo, "Experimental results for OFDM wifi-based passive bistatic radar," in 2010 IEEE Radar Conference, May 2010, pp. 516-521.

[26] L. Reichardt, C. Sturm, F. Grunhaupt, and T. Zwick, "Demonstrating the use of the IEEE 802.11p car-to-car communication standard for automotive radar," in 2012 6th European Conference on Antennas and Propagation (EUCAP), March 2012, pp. 1576-1580.

[27] Y. L. Sit, B. Nuss, S. Basak, M. Orzol, and T. Zwick, "Demonstration of interference cancellation in a multiple-user access OFDM MIMO radar-communication network using usrps," in IEEE MTT-S International Conference on Microwaves for Intelligent Mobility, May 2016, pp. 1-4.

[28] C. Aydogdu, N. Garcia, and H. Wymeersch, "Radar communication for combating mutual interference of FMCW radars," in IEEE Global Communications Conference (GlobeCom), 2018, submission awaiting decision. [Online]. Available: https://arxiv.org/abs/1807.01497

[29] M. I. Skolnik, Radar Handbook, 3rd ed. New York: McGraw-Hill Education, 2008.

[30] C. Karnfelt, A. Paden, A. Bazzi, G. E. H. Shhade, M. Abbas, and T. Chonavel, "77 GHz ACC radar simulation platform," in 9th International Conference on Intelligent Transport Systems Telecommunications, (ITST), Oct 2009, pp. 209-214.

[31] S. Lee, S. Kang, S. C. Kim, and J. E. Lee, "Radar cross section measurement with $77 \mathrm{GHz}$ automotive FMCW radar," in IEEE 27th Annual International Symposium on Personal, Indoor, and Mobile Radio Communications (PIMRC), Sept 2016, pp. 1-6. 